\title{
Reliability Analysis of Combat Architecture Model based on Complex Network
}

\author{
Xiaoguang Wang ${ }^{\text {a, }}$, Sen $\mathrm{Li}^{\mathrm{a}}$, Fuxian Liu ${ }^{\mathrm{a}}$, Xueyuan Fan ${ }^{\mathrm{a}}$ \\ ${ }^{a}$ Missile Institute, Air Force Engineering University, Sanyuan ,China
}

\begin{abstract}
Based on the complex and networking characteristics of the combat system under the informationtization condition, combining the complex network theory, the author put forwards three network extension rules and established the network-based system model. Meanwhile, this article discussed the network characteristic parameters, reliability assessment and some other problems, and further relied on the simulation to analyze the network characteristics, reliability and the vital nodes mining under the different extension rules. The research methods and conclusions in this article can be used as the combat system's arguments and references to its optimization.
\end{abstract}

Index Terms: Terms-Combat Architecture. Reliability. Complex network.Topology

(C) 2012 Published by MECS Publisher. Selection and/or peer review under responsibility of the Research Association of Modern Education and Computer Science.

\section{Introduction}

The warfare form has been proceeding from the platform-centric warfare to the network-centric warfare ${ }^{[1,2]}$, and gradually becoming the system countermeasure among the combat systems since the traditional operational command system has been not able to meet the demands of the joint operation under the information conditions. What characteristic shall the combat system to the network-centric warfare have, how about its reliability and efficiency, have been one of the hottest subjects in military field ${ }^{[1-7]}$.

Being the one of the basic theory of the complex system research, the complex network has been widely applied in the network system research, such as the society, biology and information ${ }^{[4,8]}$. The combat system has its objective laws, for example, the firepower and perception nodes are usually the end nodes, and there are strict command level structures among the nodes and the network must have the reasonable level and span structure. Therefore, the combat system network has its own characteristics in its structure and connection mechanism, and the above-mentioned network model can be not directly used in the study on combat system. Based on document $[3,5,8]$, this article further defines the network model and analyzes its reliability.

* Corresponding author.

| E-mail address: wxg0108018@163.com 


\section{Networking Description of Combat Architecture AND reliability}

\subsection{Networking Description}

Use the graph $G=(V, E)$ to depict the combat system network, here $V=\left\{v_{1}, v_{2}, \cdots v_{N}\right\}$ is the sets of $N$ nodes which correspond to each entity unit in the system; $E=\left\{e_{1}, e_{2}, \cdots e_{M}\right\}$ is the sets of $M$ edges which correspond to the interactive relations among the units, including the command control, collaboration and other interactive relations. This relation can be depicted with the network's adjacency matrix- $H=\left[h_{i j}\right]$, for instance, $h_{i j}=1$ represents that there is a certain interactive relation between node $v_{i}$ and node $v_{j}$, in contrast, $h_{i j}=0$.

The combat command needs a reasonable level relation, so the command nodes in the combat system network shall be a hierarchical organization structure, and then the author introduces the concepts of span and level here. The level is the vertical command level series symbolized with $\mathrm{D}$; span is the number of nodes governed by each level's command and control nodes symbolized with S. Combining document [3,5] to bring the following hypothesis:

(1) The top-level command nodes in the combat system is overall connectivity, viz any two top-level nodes can be directly connected.

(2) The combat system dose not exist isolated nodes, and any two nodes can realize the interaction, viz the network is a connectivity graph.

(3) The node's intrinsic attribute is determined by its belonged level. Nodes at the same level have the same index of capacity, information processing and reliability without considering the influence factors from the decision-making people, time and the environment.

According to the above hypothesis, we can do the following analyses: the network is an acyclic connected graph; since the higher level's nodes have the command control and information distribution and other interactive relations to its lower level's nodes and the lower level's nodes have the feedback and information report and other interactive relations to its higher level's nodes, so the connection edge is bidirectional, which is equivalent to undirected graph processing; the node attribute is determined by its belonged level and the edge has no weight.

\subsection{Combat Architecture Network Reliability}

The combat system network's reliability refers to: when the network is randomly fault or, disturbed, surpassed or destroyed, the capacity of the network fulfilling the warfare task. Because the network depends on nodes to realize various interactive functions and embodies its systematic feature, the connectivity is the main reliability index. Analyzing the network reliability from the angle of topology level, it expressed that the network continuously sustain its connectivity capacity after deleting some nodes or edges, which is also called the network survivability. Combining the document $[9,10]$, the reliability measurement based on the connectivity is defined as follows:

(1) Probability of Node Pair Connectivity: It is the probability that there is at least one connectivity path among nodes. The article focuses on the reliability of the network topology and does not consider the node or edge's probability of failure, here omitted.

(2) Relative Connectivity Probability. It refers to the ratio of the survived biggest number of connected sub network nodes in the number of nodes in original network when the network is randomly fault or attacked. It reflects the biggest connectivity scope the network can provide before and after the network being destroyed. 
(3) Overall Connectivity Probability.It defines that if the connectivity efficiency of the node $v_{i}$ and $v_{j}$ is the multiplicative inverse of the smallest distance, the overall connectivity probability is the average value of the connectivity probability of all nodes.

$$
E_{g}=\sum_{i \neq j} E_{i j} / N(N-1)
$$

The expression (1) demonstrated the level of difficulty of nodes connectivity. The shorter the distance of two nodes is, the higher the interactions fulfill efficiency and reliability will be. The overall connectivity efficiency reflects the network's average shortest path; which is relative to the transmission time consumption. So the document [5] takes it as a timeliness target, and the index in document [10]are the improved forms of the overall connectivity efficiency.

(4) Relative Connectivity Efficiency. It is the ratio of the connectivity efficiency of rest network produced by deleting nodes or edges local- $E_{l}$ in the overall connectivity efficiency $E_{g}$, and reflects the level of difficulty variation of the nodes trying to keep connected before and after the network being destroyed.

When the nodes and edges of combat network are fault or attacked, the network reliability is mainly manifested in the variation of the average path length and the number of nodes still relying on the rest network. Therefore, the definitions in this section are used to reflect these two aspects.

\section{Combat Architecture Network Model}

The tree combat system under the platform-centric warfare has the features of more levels, vertical long and horizontal short, and also some disadvantages, such as low efficiency, slow reaction and poor survivability, so it is an important target of the military information construction to establish the flat networkcentric warfare. After clarifying the rules of command and control, it is the inevitable choice for the system warfare to strength the information coordination capacity among nodes at the same level or different level, which will bring changes of the command level, span and form. The warfare system network in this article is divided into two parts:

\subsection{Basic Tree Network Construction}

Step1: Initiation of the number of network nodes- $N$, span $S$ and network level $D$; here $n=0$.

Step2: Get $n_{1}$ top-level nodes with $d=1$ and form an overall connectivity network; set $d=2, \quad n=n_{1}$.

Step3: Form new nodes with the corresponded level of $d$; select the nodes with the degree less than $A$ and level of $d-1$ to form a sets, then connect them with the nodes which has the shortest euclidean distance to those nodes, $n++$, come to Step 4; if the sets is null set, $d++$, come to Step 3, here if $d=2$, $A=S+n_{1}+1$, in contrast, $A=S+1$.

Step 4: if $n<N$, return to Step 3; in contract, the process ends.

Here, the number of top-level nodes- $n_{1}=N(1-S) / 1-S^{D}+1$; the formed basic network is denoted as NET.

\subsection{Basic Network Extension}

Network extension is a process of adding new nodes or edges to the original network according to some certain connection rules. The traditional tree command relation still exists, but the horizontal or cross relations among the warfare units at the same level or different level become more prominent, thus ensuring the more 
flexible command mode, more convenient coordination capacity and higher survivability and restructure capacity. Therefore, the combat system network not only needs a clear command relation, but also a stronger coordination capacity at the same level or different levels. In order to analyze the influences of the extension rules on the warfare network, the network extension evolution rules are determined as follows:

Rule A: Randomly select the nodes to be extended with the corresponded level of $d$.Seek the node sets of the nodes with level of $[d-1, d+1]$ within the radius of $R$, connect the nodes which has the shortest Euclidean distance from the extended nodes, and finally get the network symbolized with NET-D.

Rule B: Randomly select the nodes to be extended with the corresponded level of $d$, seek the sets of nodes with the level of $[d-1, d+1]$ and depend on probability $p$ to select one of them for connection and get a network symbolized with NET-BA.

$$
p=K_{a} / \sum_{b} K_{b}
$$

Here, $K_{a}$ is the degree of selectable nodes; $\sum_{b} K_{b}$ is the sum of degrees of the node sets $\sum_{b} K_{b}$. Rule B is the improved form of scale-free network's preferential evolution rules.

Rule C: Based on Rule B, adding another condition that the seeking radius of $R$ to get the network symbolized with NET-BAD. Here, provided that the network distribution is normalized to 1, the number of top-level nodes is $n_{1}$, then $R=1 / 2 n_{1}$.

\section{Experimental Analysis}

According to the methods mentioned in section 4, if $N=300 、 S=6 、 D=4$, it can randomly form the NET network as shown in Figure1. Then, according to the Rule A, B and C, extend the NET network 100 times to get networks symbolized with NET-D、NET-BA and NET-BAD.

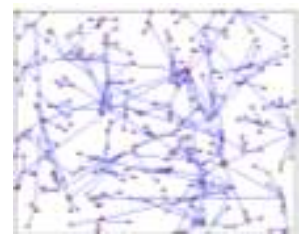

(a)

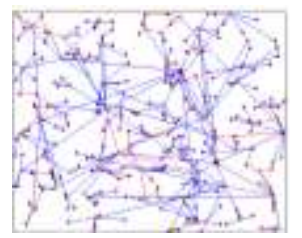

(b)

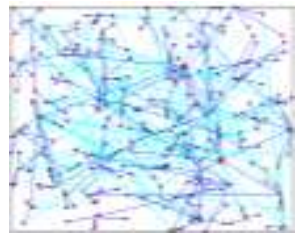

(c)

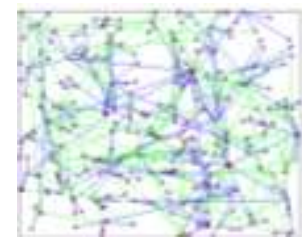

(d)

Fig.1. (a) NET, (b) NET-D, (c) NET-BA, (d) NET-BAD

\subsection{Analysis on Network Characteristic Parameters}

Firstly, let's discuss the characteristics of the degree distribution. The network degree distribution is shown as Figure 5 with the same corresponding relation of Fig 1. The solid line represents the fitted power law curve and the point represents the actual degree distribution. It is clearly seen that the NET and NET-D have an uneven degree distribution with unobvious power law characteristic; but the NET-BA and NET-BAD have obvious power law characteristics with the scale factor $r$ of 1.89 and 1.85 respectively and both of them are scale-free network. 

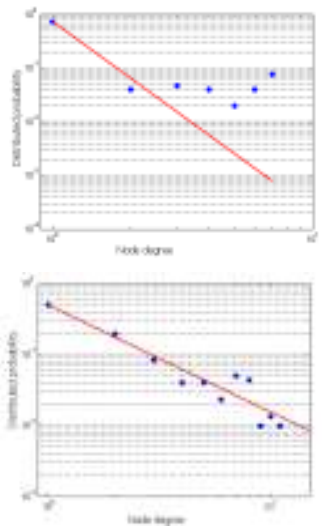
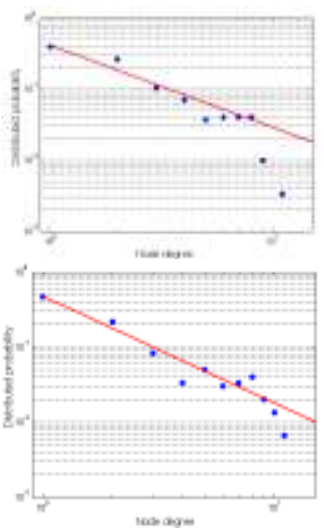

Fig. 2. Degree Distribution and Fitting of Networks

Then, let's discuss the clustering coefficient and average path length. Since the basic network NET is a tree structure and there are no triangle structures formed among its nodes, the clustering coefficient of all the nodes are 0. As shown in Figure6, NET-D has a bigger clustering coefficient, especially the rearmost-numbered end nodes Those end nodes have smaller original degrees and easily form a small-scale community structure situated in the network end if Rule A uses the connection choose-closed principle.. The newly-added connections in NET-D are always the neighboring nodes in topology, so it has a slight influence on the distance among nodes and the average path length changes little(see Figure7). In summary, NET-D is more suitable for the warfare network's local information sharing and coordination warfare. NET-BA uses the overall choose-closed principle, so it easily forms high degree nodes but the nodes have smaller clustering coefficient and unobvious community structure. NET-BAD uses the local preferential principle under the path length limitation to form various nodes with high clustering coefficient various middle-scaled communities. Meanwhile, the communities of NET-BA and NET-BAD are basically formed by the higher-level nodes in the basic network with the shorter average path length, so they are more convenient for the warfare network to perform the overall information sharing and large-scaled coordination warfare.

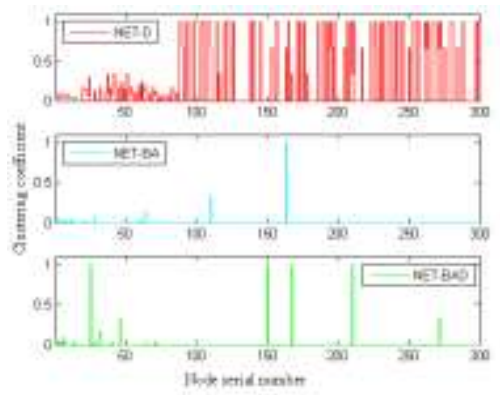

Fig. 3. Clustering Coefficient of Nodes 


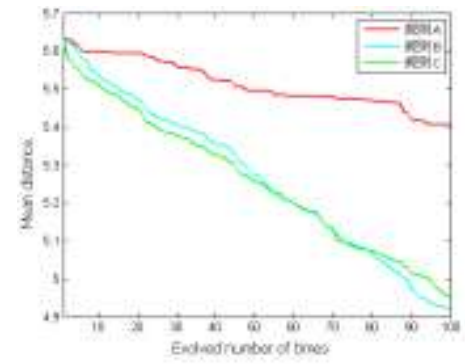

Fig. 4. Extension Times and Average Path Length

\subsection{Reliability Analysis on Combat Network}

Based on the different modes of removing nodes, the simulation can be divided into two parts: random failure (fault) and deliberate attack. The deliberate attack also has several strategies, which presents the attack order decided by the importance. The different importance assessment will result in the different attack modes, like the attack strategy based on nodes degree values or betweeness. This section selects the deliberate attack based on degree value for simulation.

Seeing from Figure8 and 9, the reduction of relative connectivity probability caused by deliberate attack is more rapid than the reduction caused by random failure. Taking the NET-BA as an example, when the fault nodes reach to $5 \%$ of the total, the whole network collapses; by contract, when the random failure reaches to $70 \%$, the network basically collapses. Because of the addition of connection edges, the extended network has a higher reliability. Meanwhile, both NET-BA and NET-BAD are scale-free network, and have a stronger flexibility to the accidental failure, so they are more reliable than NET-D.

In the first phase of failure, before the network division (except for NET network because of the tree structure), the relative connectivity probability of deliberate attack is a little lower than the probability of random failure. The reason is that the original failure nodes caused by deliberate attack are

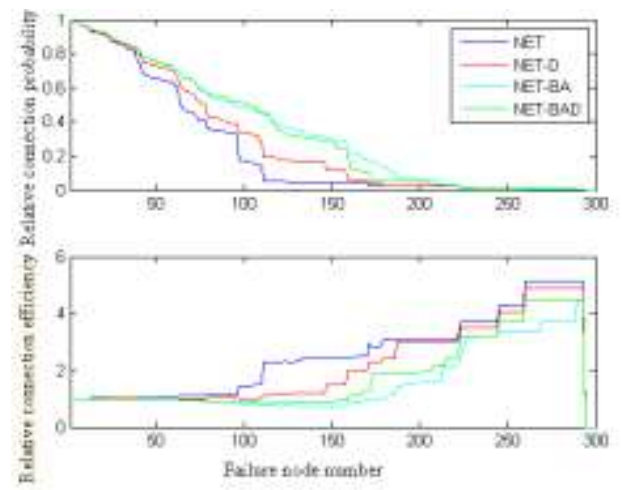

Fig. 5. Network Reliability under the Random Failure 


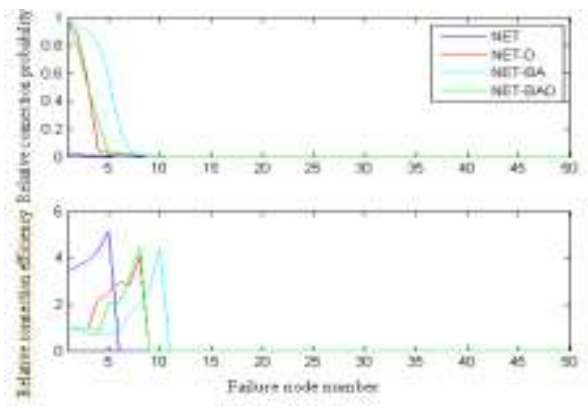

Fig. 6. Network Reliability under the Intensive Attack

always from the top-level command nodes and information integration center, so it will cause various failure of the shortest paths and make the network have to choose the relative shorter paths, which will increase the network average path length and decrease the transmission efficiency. With the failure nodes increasing, the relative connectivity efficiency rapidly increases and immediately returns to zero after reaching to a crest value, and the reason is that the network is split into several sub networks, the scale is downsizing and network average path length is reducing and even reaches to zero, then the network collapses. In the course of this process, the network collapse caused by deliberate attack is faster than the collapse caused by random failure.

In conclusion, the network extension strengths the warfare network's same level or cross level coordination, effectively improves the command coordination efficiency and reliability of the warfare system. Meanwhile, different extension rules can produce different network topology and also present different network statistic characteristics and reliability.

\section{Conclusions}

This article combines the complex network theory to primarily discuss the combat system network's topology characteristics, model and reliability, but it also needs a further study. For example, as shown in Fig 1.3: the extension edges of the network extension Rule B have bigger Euclidean distance, which means a higher construction cost, but we can further narrow the distance limitation $R$ in Rule $\mathrm{C}$ to solve it; meanwhile, all warfare network construction needs considering more actual factors, like the cost, distribution, functions and so on, and it can not use the model in the article to do the concrete case analysis. This article's model and analysis method can provide reference and basic conclusion to define the practical problems.

\section{References}

[1] Jinjun Li, Shan Yi and Louyang Li, “Analysis Method and Model of Combat Architecture on Theory of Complex Networks[J],”Journal of System Simulation, ,vol. 19,no. 2,pp. 151-154, February 2007.

[2] Chunli Li, Yilai Chen, “Intergrated Joint Operation Command[M],"Bei Jing:Military Science Press,2004.

[3] Tao Zhu, Guocen Chang and Shuiping Zhang,"Research on Model of Command and Control Information

Cooperation Based on Complex Networks[J],’Journal of System Simulation, vol. 20,no. 22,pp.6058-6060, November 2008.

[4] Deyi Li, Xinzheng Wang and Gangfeng Hu,"Networking Warfare and Complex Networks[J],"Chinese Military Science,vol.19,no.3,pp.111-119, March 2006.

[5] Jinjun Li, Guoguang Liu and Huang Qian,"Analysis Method and Model of Combat Command Architecture Based on Theory of Complex Networks[J], ’Journal of System Simulation, vol. 20,no. 17,pp. 
4712-4715, September 2008.

[6] Lina Chen, Jincai Huang and Weiming Zhang,"Research on Complex Network Topology Model of Network Warfare[J],” Electronics Optics \& Control, vol. 15,no. 6,pp. 4-6. June 2008.

[7] Mingke Zhang, Zheng Chen and Changjun Yu,"Complex Networks Topology Modeling in Network Warfare[J],"Aerospace Control, vol. 25,no. 4,pp. 3-6. October 2007.

[8] Weixin Jin, Tianyuan Xiao,"Study on Combat Architecture Based on Complex Networks[J],"Complex Systems and Complexity Science, vol. 6,no. 4,pp. 12-25. September 2009.

[9] Jun Wu, Yuejin Tan,"Study on Measure of Complex Networks Invulnerability[J],"Journal of Systems Engineering,vol. 20,no. 2,pp. 128-131, April 2005.

[10] Ming ke Zhang, Changjun Yu and Qiang Yang."Study on Invulnerability of Information Fusion Networks Based on Network Dynamics[J],"Systems Engineering and Electronics, vol. 30,no. 12,pp. 24212424. April 2008. 